\title{
Bone Marrow Mesenchymal Stem Cells Transfected with Distal-Less Homeobox 2 Gene Promote Articular Cartilage Repair after Injury
}

\author{
C. YANG, Y. CHEN AND YUAN YUAN*
}

Department of Orthopedics, Hwa Mei Hospital, University of Chinese Academy of Sciences, No. 41 Xibei Road, Ningbo, Zhejiang 315000, China

\author{
Yang et al.: Bone Marrow Mesenchymal Stem Cells Transfected with Distal-Less Homeobox 2 Gene
}

\begin{abstract}
To investigate the repair effect of distal-less homeobox 2 transfected bone marrow-derived mesenchymal stem cells on knee cartilage injury. 20 male New Zealand large eared rabbits aged 6 to 7 mo were selected and fed for $1 \mathrm{w}$ to undergo knee cartilage injury modeling surgery. Reverse transcription-polymerase chain reaction, western blot and hematoxylin-eosin staining were used to detect these related indicators. Behavioral scores and cartilage repair in each group of large rabbits was calculated. The Wakitani total score showed a very significant level of improvement in the repair of knee cartilage damage in New Zealand rabbits in the distal-less homeobox 2 transfected and bone marrow mesenchymal stem cells groups $(\mathbf{p}<0.01)$. Reverse transcription-polymerase chain reaction results showed that after 4 and $8 \mathrm{w}$ intervention, the messenger RNA levels were significantly increased $(\mathbf{p}<0.01)$. Western blot results showed that 4 and 8 w after surgery, the expression of type I collagen, type II collagen and proteoglycan aggrecan proteins were significantly increased. The injection of simple bone marrow mesenchymal stem cells could promote the repair of cartilage damage and the distal-less homeobox 2 transfected mesenchymal stem cells had the best repair effect after 8 $w$, indicating that the distal-less homeobox 2 gene could regulate the proliferation and differentiation of bone marrow mesenchymal stem cells and promote the repair of damage in a long period of time.
\end{abstract}

Key words: Bone marrow-derived mesenchymal stem cells, distal-less homeobox 2, articular cartilage injury

Cartilage injury, as one of the common sports injury diseases, occurs in all articular cartilage of the whole body and knee joint cartilage injury is the most common $^{[1,2]}$. Knee joint cartilage damage is also known as knee joint degeneration, knee osteoarthritis, etc. ${ }^{[3,4]}$, is mainly due to the partial destruction or degeneration of articular cartilage, leading to knee joint dysfunction or even loss of activity, mainly manifested as swelling and pain around the knee joint, dyskinesia, walking difficulty and aggravating pain after strenuous exercise. If the injury is not treated effectively, the condition will further aggravate and eventually lead to the complete loss of knee joint function.

At present, a large number of clinical treatment methods for knee joint cartilage injury, including massage, acupuncture and hot compress of traditional Chinese medicine, are widely used in clinical practice. Although they could effectively relieve local pain of knee joint in patients in a short period of time, play the role of promoting blood circulation and removing blood stasis, promoting qi and relieving pain, they can only temporarily improve the symptoms of knee joint discomfort and cannot completely cure the disease ${ }^{[5]}$. In addition, many other methods, such as drug injection of anti-inflammatory agents and sodium hyaluronate, can reduce the inflammatory reaction of the lesion site, eliminate inflammatory factors and alleviate the patient's condition. If the patient's condition is serious, arthroscopic surgery, minimally invasive surgery or knee arthroplasty can be used for treatment ${ }^{[6,7]}$. However, the cost of modern medical treatment is very high, the side effects are large and the condition cannot be completely controlled.

Recent studies have found that bone marrow mesenchymal stem cells (BMSCs) can differentiate into osteoblasts and chondrocytes, which can promote cartilage repair ${ }^{[7-9]}$. Distal-less homeobox 2 (DLX2) gene, belonging to the DLX protein family, is a

*Address for correspondence

E-mail: sky.6228@163.com 
key transcription factor that can induce BMSCs to differentiate into chondroblasts and osteoblasts and plays an important role in the process of bone repair and reconstruction. This study aims to explore the regulatory effect of DLX2 gene on cartilage repair of knee joint by constructing a New Zealand rabbit knee articular cartilage injury model and further clarify the role of DLX2 gene in the field of cartilage damage repair, so as to provide more treatment options for patients with knee joint cartilage injury.

\section{MATERIALS AND METHODS}

\section{Raising experimental animals:}

A total of 20 male New Zealand rabbits aged 6-7 mo, weighing $2.5-2.7 \mathrm{~kg}$ (from the experimental animal research center of Sun Yat-sen University) were selected and fed in the experimental animal center of our hospital. The total duration of the experiment was 9 w. After $1 \mathrm{w}$ of adaptive feeding, the knee joint cartilage injury model was established and samples were taken at $4 \mathrm{w}$ and $8 \mathrm{w}$ respectively. All the experimental animals were given sufficient feed and water. Feed and water were added regularly every day. The laboratory animal room was cleaned and the excreta were treated. The animal room is equipped with air conditioning, exhaust fan and other necessary equipment to ensure normal ventilation and ventilation throughout the day. The indoor temperature is controlled at $24 \pm 2^{\circ}$ and the humidity is $40 \%-70 \%$.

\section{Extraction and culture of bone marrow mesenchymal cells:}

1 mo old New Zealand rabbit, weighing about $0.3 \mathrm{~kg}$, was put into $75 \%$ alcohol container for 15 min after decapitation and then aseptic operation was carried out in ultraclean table. Bilateral tibia, femur and humerus were removed and the attached muscle and ligament tissues were removed and then washed twice with normal saline. In a sterile environment, the long bone was cut short and the serum Dulbecco's modified eagle medium (DMEM) without blood glucose was drawn with a $10 \mathrm{ml}$ syringe and repeatedly washed until the bone turned white. Then, the single cell suspension was made by repeatedly aspirating with a needle. After being put into a centrifuge tube, it was centrifuged at $1000 \mathrm{rpm}$ for $5 \mathrm{~min}$. The supernatant was discarded, the cell precipitate was resuspended and inoculated in the culture medium containing $15 \%$ fetal bovine serum (FBS).

\section{Establishment of articular cartilage injury model:}

About $2.6 \mathrm{~kg}$ male New Zealand rabbits were used as the model objects and $20 \%$ urethane solution was prepared for ear edge intravenous injection anesthesia at $5 \mathrm{mg} /$ $\mathrm{kg}$. After anesthesia, the limbs of the experimental animals were fixed on the operating table and the operation area was disinfected with iodophor for 3 times. A transverse incision was made on the lateral side of both knee joints and the incision length was about 2 $\mathrm{cm}$. The medial articular cavity and medial meniscus can be clearly observed and articular cartilage can be fully exposed. Defects of $2 \mathrm{~mm} \times 4 \mathrm{~mm} \times 1 \mathrm{~mm}$ are made on articular cartilage surface with Kirschner wire and then washed with normal saline. Put them into the $26^{\circ}$ incubator and put them back into the animal room after the animals wake up from anesthesia, continuous $5 \mathrm{~d}$ of intramuscular injection of $2-3 \mu / \mathrm{kg}$ penicillin sodium to prevent wound infection.

\section{Animal grouping:}

According to the principle of random control, the experimental animals were randomly divided into control group $(n=5)$, sodium hyaluronate group $(n=5)$, BMSCs group $(n=5)$ and DLX2 transfection group $(\mathrm{n}=5)$. The treatment methods of New Zealand rabbits in each group were as follows. Control group: After modeling, $2 \mathrm{ml}$ normal saline was injected into articular cavity; sodium hyaluronate group: after modeling, 2 $\mathrm{ml} 1 \%$ sodium hyaluronate was injected into articular cavity; BMSCs group: after modeling, $2 \mathrm{ml} 1 \%$ sodium hyaluronate solution was injected into articular cavity; BMSCs group: After modeling, $2 \mathrm{ml}$ normal saline containing $1 \times 107$ units of pure BMSCs was injected into the articular cavity. In DLX2 transfection group, $2 \mathrm{ml}$ of normal saline containing $1 \times 107$ units of DLX2 transfected BMSCs was injected into the articular cavity after modeling.

\section{Hematoxylin-eosin (HE) immunofluorescence staining:}

At the $4^{\text {th }}$ and $8^{\text {th }} \mathrm{W}$ after operation, the cartilage defect tissues were cleaned with phosphate buffered saline (PBS) solution, soaked in $4 \%$ paraformaldehyde and fixed for $24 \mathrm{~h}$ under the condition of avoiding light and then washed with pure water for $1 \mathrm{~d}$. After conventional paraffin embedding and sectioning, the sections were observed under the electron microscope.

\section{Western blot analysis:}

The rabbit bone tissue was cut into pieces and treated with radioimmunoprecipitation assay buffer (RIPA) 
solution. The extracted protein was quantified by the biconcanilic acid kit. A total of $30 \mu \mathrm{g}$ protein samples were isolated by $10 \%$ sodium dodecyl sulfate polyacrylamide gel electrophoresis (SDS-PAGE) and then transferred to polyvinylidene fluoride (PVDF) membrane. After blocking with tris-buffered saline (TBS) of $5 \%$ bovine serum albumin (BSA) $+0.1 \%$ Tween 20 for $1 \mathrm{~h}$, the membrane was incubated with anti DLX2 (1:500) overnight at $4^{\circ}$. Then the goat anti mouse secondary antibody (1:1000) coupled with horseradish peroxidase was incubated for $1 \mathrm{~h}$. Fluorescence imaging system was used to analyze the results. The density of protein bands was analyzed by Image $\mathrm{J}$ software.

\section{Reverse transcription-polymerase chain reaction (RT-PCR) analysis:}

Trizol reagent was used to extract total ribonucleic acid (RNA) from rabbit cartilage. Use PrimeScript according to the manufacturer's instructions, gamma RT kit reverse transcripted $5 \mu \mathrm{g}$ total RNA into complementary DNA (cDNA). SYBR green kit was used to detect the messenger RNA (mRNA) expression level of specific primers and $2^{-\Delta \Delta \mathrm{CT}}$ method was used to evaluate. The thermal cycling conditions are as follows: $5 \mathrm{~min}$ at $95^{\circ}, 30 \mathrm{~s}$ at $95^{\circ}, 30 \mathrm{~s}$ at $57^{\circ}$ and $1 \mathrm{~min}$ at $72^{\circ}$ for 4-5 cycles. Gene specific primers were synthesized by Thermo Fisher technologies. All primer sequences are shown in Table 1.

TABLE 1: THE PRIMER SEQUENCES USED FOR RT-PCR IN THIS STUDY

\begin{tabular}{lc}
\hline Gene & \multicolumn{1}{c}{ Sequences } \\
\hline Col-II & F: TGCAGGAGGGGAAGAGGTAT \\
& R: GGCAGTCCTTGGTGTCTTCA \\
AGG & F: GAGGTGGTGGTGAAGGTGT \\
& R: GTGTGGATGGGGTACCTGAC \\
Col-I & R: CCCAGGTCCTCAGGGTCTTC \\
& F: GACCACCCAGCCGAACTT \\
DLX2 & R: CCGCCATGACAGTAACCAC \\
Glyceraldehyde & F: CTGGGCTACACTGAGCACC \\
3-phosphate & R: AAGTGGTCGTTGAGGGCAATG \\
\hline
\end{tabular}

\section{Cartilage repair score:}

The animals in each group were free to move in the cage for $2 \mathrm{w}$ after modeling. The training was started at the $3^{\text {rd }} \mathrm{W}$ after the operation and the moderate intensity horizontal treadmill exercise $(12 \mathrm{~m} / \mathrm{min}$, lasting for $30 \mathrm{~min}$ ) was used. After $8 \mathrm{w}$ of training, the rats were killed and the histological evaluation of cartilage repair was performed. The Wakitani cartilage repair scoring system was used to evaluate the repair of cartilage ${ }^{[19]}$.

\section{Behavioral score:}

Basso, Beattie, Bresnahan (BBB) scoring method was used to evaluate the motor function of bilateral lower limbs of New Zealand rabbits in single blind condition. The scores were scored on the $1^{\text {st }} \mathrm{d}$, the $1^{\text {st }} \mathrm{W}$, the $2^{\text {nd }} \mathrm{W}$ and the $3^{\text {rd }} \mathrm{W}$.

\section{Statistics and analysis:}

All data were analyzed and processed by statistical package for the social sciences (SPSS) 21.0 and Graphpad Prism 8.0. The significant differences among the related indexes were analyzed by sample $t$ test and analysis of variance (ANOVA) test. The data analyzed and processed were expressed as mean \pm standard deviation $(x \pm s), p<0.05, p<0.01$ means that the data have significant difference.

\section{RESULTS AND DISCUSSION}

The results demonstrated that the motor function of the animals in each group disappeared completely and the $\mathrm{BBB}$ score was 0 , indicating that the model was successfully established. In the same treatment group, there were significant differences in scores at different time points $(\mathrm{F}=1762.323, \mathrm{p}<0.05)$. As shown in fig. 1, with the prolongation of treatment time, the BBB scores of mice in each group showed an increasing trend and the total score difference between different treatment groups was statistically significant $(\mathrm{F}=211.289$, $\mathrm{p}<0.05)$. The results showed that the BBB score of DLX2 transfection group was the highest at the same time, which had no significant difference with BMSCs group ( $p>0.05)$, but was significantly higher than the other two groups $(\mathrm{p}<0.05)$.

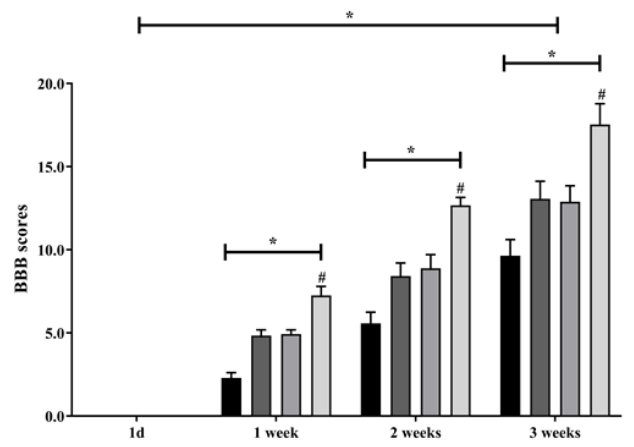

Fig. 1: Behavioral scoring results of each group Note: ${ }^{*} \mathbf{p}<0.05$, compared with trend groups; ${ }^{\# p}<0.05$, compared with control and sodium hyaluronate groups. ( $($ ) Control; (ロ) Sodium hyaluronate; (ロ BMSCs; (口) DLX2+BMSCs

Cartilage repair score results were calculated. The 
results showed that compared with the control group, the other three groups of New Zealand rabbit cartilage injury in cell morphology, matrix staining intensity, surface regularity, cartilage thickness, neighboring organization integration and Wakitani cartilage damage were repaired and improved significantly $(p<0.05)$, among which DLX2 transfection group and BMSCs group had the highest function improvement $(\mathrm{p}<0.01)$, as shown in fig. 2 .

Expression level of type I collagen (Col-I) mRNA was also observed. At $4 \mathrm{w}$ after operation, the expression of Col-I mRNA in each experimental group was markedly higher than that in control group $(\mathrm{p}<0.01)$ and the expression level of Col-I mRNA in DLX2 transfection group was significantly lower than that in sodium hyaluronate group $(\mathrm{p}<0.05)$, but higher than that in BMSCs group $(\mathrm{p}<0.05)$. At $8 \mathrm{w}$ after operation, the expression of Col-I mRNA in each experimental group was significantly higher than that in control group $(p<0.01)$. The expression of Col-I mRNA in DLX2 transfection group was significantly lower than that in sodium hyaluronate group $(\mathrm{p}<0.05)$, but there was no significant difference between DLX2 transfection group and BMSC group ( $\mathrm{p}>0.05)$, as shown in fig. 3 .

Expression of type II collagen (Col-II) mRNA was also observed. At $4 \mathrm{w}$ after operation, the expression of Col-II mRNA in each experimental group was significantly higher than that in control group $(\mathrm{p}<0.01)$ and the DLX2 transfection group was significantly higher than in BMSCs group $(\mathrm{p}<0.05)$, but there was no significant difference between DLX2 transfection group and sodium hyaluronate group ( $\mathrm{p}>0.05)$. At $8 \mathrm{w}$ after operation, the expression level of Col-II mRNA in each experimental group was significantly higher than that in control group $(\mathrm{p}<0.01)$ and that in DLX2 transfection group was significantly higher than that in sodium hyaluronate group and BMSCs group $(\mathrm{p}<0.05)$, as shown in fig. 4.

The expression level of proteoglycan aggrecan (AGG) mRNA was determined. At $4 \mathrm{w}$ after operation, the expression of AGG mRNA in each experimental group was obviously higher than that in control group $(p<0.01)$. DLX2 transfection group was significantly higher than in BMSCs group $(\mathrm{p}<0.05)$, but there was no significant difference between DLX2 transfection group and sodium hyaluronate group $(\mathrm{p}>0.05)$. At $8 \mathrm{w}$ after operation, DLX2 transfection group and sodium hyaluronate group were markedly higher than that in control group $(p<0.01)$, there was no significant difference between BMSCs group and control group $(\mathrm{p}>0.05)$ and DLX 2 transfection group was significantly higher than sodium hyaluronate group and BMSCs group ( $\mathrm{p}<0.05)$, as shown in fig. 5 .

Compared with the control group, the expression of Col-I, Col-II and AGG protein in each experimental group was significantly increased $(p<0.05)$, the expression of Col-II and AGG in DLX2 transfection group was significantly higher than that of other experimental groups $(\mathrm{p}<0.05)$ and the expression of Col-I in DLX2 transfection group was significantly higher than that in BMSCs group, but there was no significant difference between DLX2 transfection group and sodium hyaluronate group $(\mathrm{p}>0.05)$, as shown in fig. 6 .
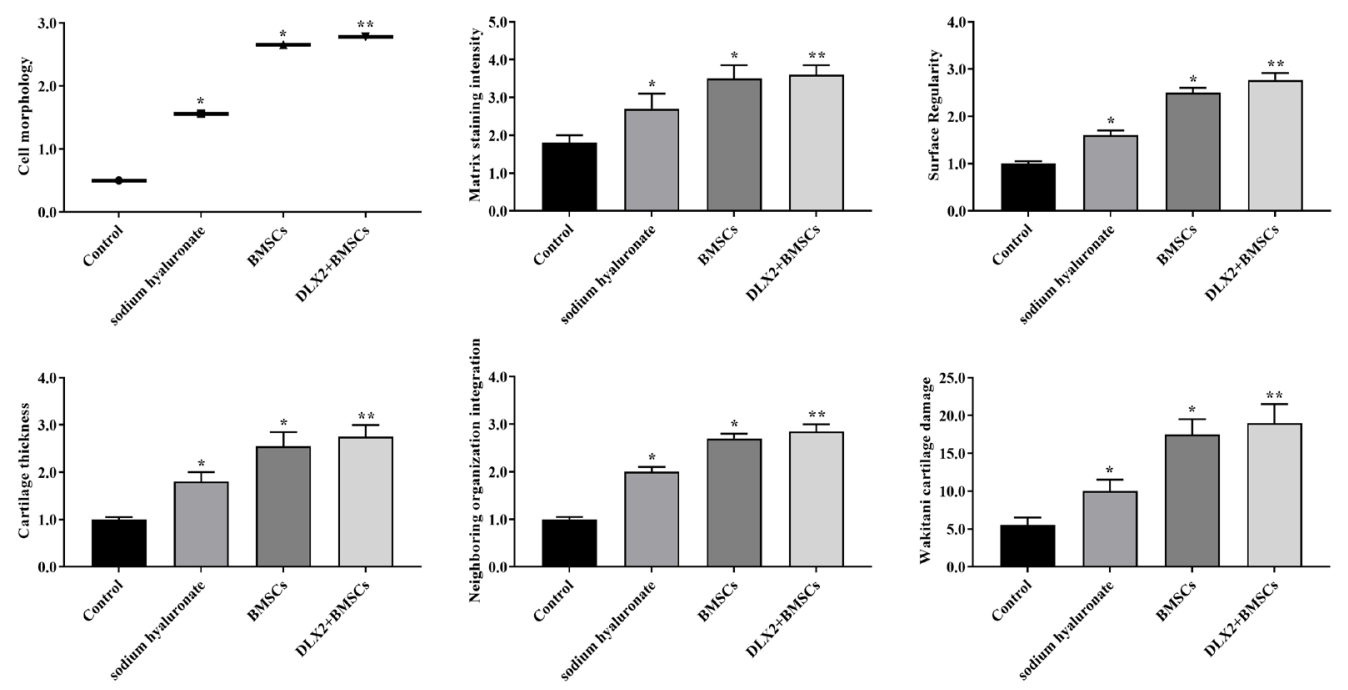

Fig. 2: Cartilage repair score results

Note: ${ }^{*} \mathbf{p}<0.05, * * p<0.01$, compared with control group 


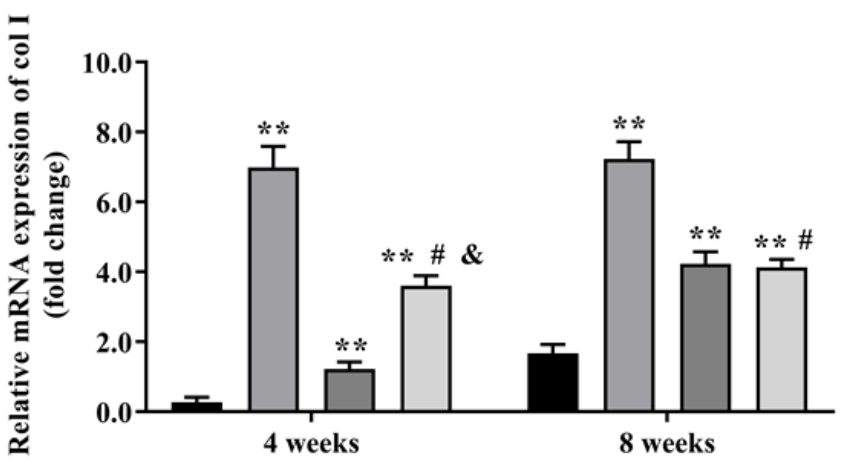

Fig. 3: The expression level of Col-I mRNA

Note: ${ }^{* *} \mathbf{p}<0.01$, compared with control group; ${ }^{*} \mathbf{p}<0.05$, compared with sodium hyaluronate group; \&p<0.05, com-

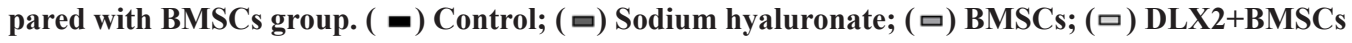

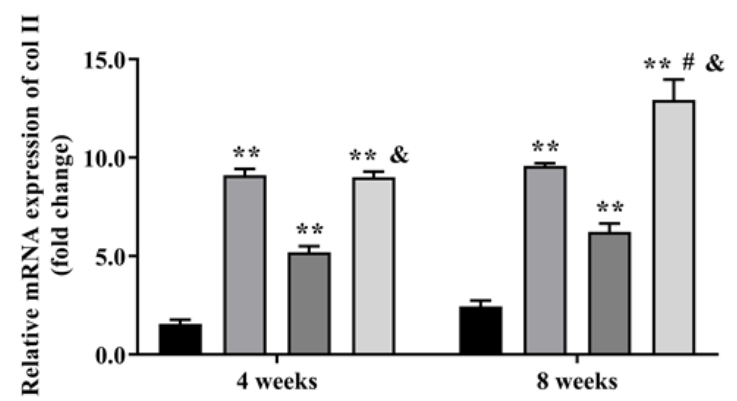

Fig. 4: The expression level of Col-II mRNA

Note: ${ }^{* *} \mathbf{p}<0.01$, compared with control group; ${ }^{*} \mathbf{p}<0.05$, compared with sodium hyaluronate group; \&p<0.05, com-

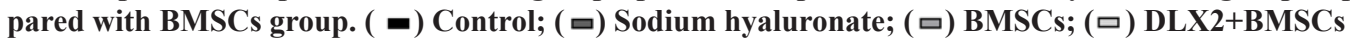

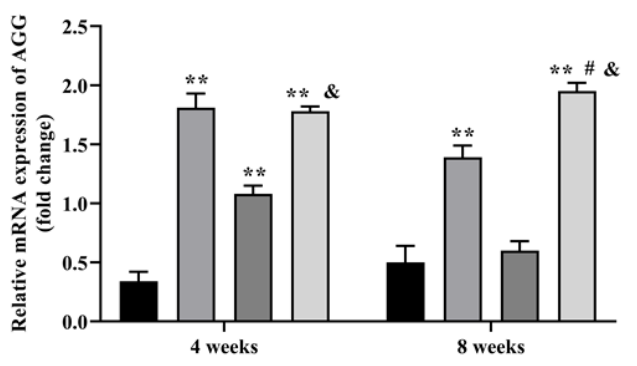

Fig. 5: The expression level of AGG mRNA

Note: **p<0.01, compared with control group; ${ }^{*} \mathbf{p}<0.05$, compared with sodium hyaluronate group; \&p<0.05, compared with BMSCs group. ( ש) Control; (ם) Sodium hyaluronate; (ם) BMSCs; (ם) DLX2+BMSCs
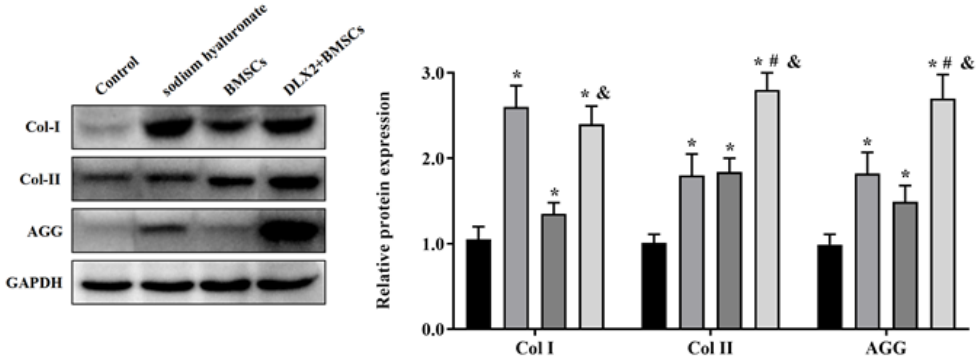

Fig. 6: The expression of related proteins

Note: *p<0.05, compared with control group; ${ }^{\#} \mathbf{p}<0.05$, compared with sodium hyaluronate group; $\& \mathbf{p}<0.05$, com-

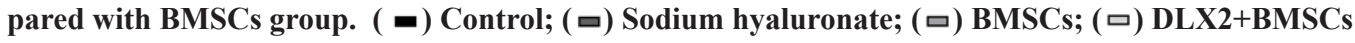


With the popularization of national fitness and the influence of aging population, articular cartilage injury is particularly common in clinical practice and the proportion of knee joint cartilage damage is the highest ${ }^{[10-13]}$. The main causes of injury include wear caused by long-term high intensity load of knee joint, injury and degenerative changes caused by violent traction. Because of its own structural factors, the ability of cartilage self-healing is very poor. At present, in addition to autologous cartilage transplantation, the research of repairing cartilage injury with autologous or allogeneic MSCs is also a treatment prospect ${ }^{[13-15]}$. BMSCs derived from bone marrow have good ability of paracrine and redifferentiation of osteoblast growth factor. In many animal experiments and even human clinical trials, BMSCs have shown certain positive effects and potential clinical application prospects in the repair of bone and joint injury ${ }^{[16-18]}$. In this study, a New Zealand rabbit model of knee cartilage injury was established and normal saline, sodium hyaluronate, BMSCs alone or DLX2 were injected into the articular cavity of each group of experimental animals to transfect BMSCs. Through histological observation, western blot analysis, RT-PCR detection and histopathological staining, the regulatory effect of DLX2 gene on articular cartilage repair was investigated.

DLX2 gene, a member of DLX transcription factor family, is an important transcription factor in bone tissue development. It can regulate the differentiation of BMSCs into osteoblasts and chondrocytes ${ }^{[19]}$. DLX2 is mainly involved in the proliferation and differentiation of osteoblasts and chondrocytes. AGG, Col-I and ColII are important components of the extracellular matrix of cartilage, accounting for more than $90 \%$ of the extracellular matrix. AGG in normal cartilage tissue contains a large number of hydrophilic groups, which can contain a lot of water, so as to keep good elasticity and compression resistance of articular cartilage and can bear the huge pressure of body weight, which plays an important role in the maintenance of joint function. Col-I and Col-II are the most abundant fibroproteins in articular cartilage extracellular matrix, accounting for $10 \%-20 \%$ of the wet weight of articular cartilage and are the specific collagen of articular cartilage ${ }^{[20]}$. Through the interaction with type IX and XI cartilage collagen, three-dimensional fiber network is formed to provide tensile strength for cartilage, promote cartilage formation and maintain soft normal function. In this study, the mRNA and protein levels of AGG, Col-I and Col-II were detected by RT-PCR and western blot techniques respectively. The results showed that the mRNA and protein expression levels of AGG in sodium hyaluronate group, BMSCs group and DLX2 group were significantly higher than those in control group. DLX2 transfection group was significantly higher than that in BMSCs group, but there was no significant difference between DLX2 transfection group and sodium hyaluronate group. Previous studies have shown that the high expression of DLX2 in hypertrophic cartilage and hypertrophic cartilage can promote the hypertrophy of chondrocytes and the process of endochondral ossification and stabilize the phenotype of chondrocytes ${ }^{[21-23]}$. Endochondral ossification is an important link in the growth and development of bone tissue and the hypertrophy and degeneration of chondrocytes is the precondition of this endochondral ossification. In the process of chondrocyte differentiation, DLX2 gene can regulate AGG, Col-I and Col-II, induce vascular invasion, thus promoting the process of cartilage ossification. In the experimental study of DLX2 knockout mice, it was found that the maturation of mouse chondrocytes was inhibited and the number of hypertrophic chondrocytes expressing Col-II was significantly reduced.

This study compared the effect of BMSCs and DLX2 from multiple levels. The results also indirectly proved that the effect of BMSCs on cartilage repair was not strong enough; compared with short-term sodium hyaluronate, the gene expression of BMSCs promoting cartilage repair was far from enough; compared with long term DLX2 transfection group, BMSCs still had the ability to repair cartilage. However, it was not as good as DLX2 transfection group. In addition, to HE staining, we can find that although DLX2 is not as good as sodium hyaluronate in the repair of cartilage injury, but BMSCs repair is better than hyaluronic acid treatment group and the cell arrangement is neat, the cartilage surface is more uniform, can also better repair cartilage. However, although sodium hyaluronate can repair cartilage injury in a short time, the cell arrangement is disordered and the surface of hose is rough. After a long time, the cartilage damage of knee joint may be further deteriorated.

In conclusion, BMSCs injected alone can promote the repair of cartilage injury to a certain extent, while the BMSCs transfected with DLX2 gene have the best repair effect after $8 \mathrm{w}$, which indicates that DLX2 gene can regulate the proliferation and differentiation of BMSCs in a long time, so as to promote the repair of injury. 


\section{Acknowledgements:}

This work was supported by the Medical Scientific Research Foundation of Zhejiang Province, China (Grant No.2021ZH035).

\section{Conflicts of interest:}

The authors report no conflicts of interest.

\section{REFERENCES}

1. Harris SE, Guo D, Harris MA, Krishnaswamy A, Lichtler A. Transcriptional regulation of BMP-2 activated genes in osteoblasts using gene expression microarray analysis: role of Dlx2 and Dlx5 transcription factors. Front Biosci 2003;8(4):1249-65.

2. Ferguson CA, Tucker AS, Sharpe PT. Temporospatial cell interactions regulating mandibular and maxillary arch patterning. Development 2000;127(2):403-12.

3. Zhou Z, Apte SS, Soininen R, Cao R, Baaklini GY, Rauser RW, et al. Impaired endochondral ossification and angiogenesis in mice deficient in membrane-type matrix metalloproteinase I. Proc Natl Acad Sci USA 2000;97(8):4052-7.

4. Ijiri K, Zerbini LF, Peng H, Correa RG, Lu B, Walsh N, et al. A novel role for GADD45 $\beta$ as a mediator of MMP-13 gene expression during chondrocyte terminal differentiation. J Biol Chem 2005;280(46):38544-55.

5. Bendall AJ, Hu G, Levi G, Abate-Shen C. Dlx5 regulates chondrocyte differentiation at multiple stages. Int J Dev Biol 2003;47(5):335-44.

6. Ferrari D, Kosher RA. Dlx5 is a positive regulator of chondrocyte differentiation during endochondral ossification. Dev Biol 2002;252(2):257-70.

7. Dai J, Kuang Y, Fang B, Gong H, Lu S, Mou Z, et al. The effect of overexpression of Dlx2 on the migration, proliferation and osteogenic differentiation of cranial neural crest stem cells. Biomaterials 2013;34(8):1898-910.

8. Takaishi H, Kimura T, Dalal S, Okada Y, D'Armiento J. Joint diseases and matrix metalloproteinases: a role for MMP-13. Curr Pharm Biotechnol 2008;9(1):47-54.

9. Liu Z, Tang Y, Qiu T, Cao X, Clemens TL. A dishevelled-1/ Smad1 interaction couples WNT and bone morphogenetic protein signaling pathways in uncommitted bone marrow stromal cells. J Biol Chem 2006;281(25):17156-63.

10. Iwamoto T, Nakamura T, Doyle A, Ishikawa M, De Vega S, Fukumoto S, et al. Pannexin 3 regulates intracellular ATP/ cAMP levels and promotes chondrocyte differentiation. J Biol Chem 2010;285(24):18948-58.

11. Kim YJ, Lee MH, Wozney JM, Cho JY, Ryoo HM. Bone morphogenetic protein-2-induced alkaline phosphatase expression is stimulated by Dlx 5 and repressed by Msx2. J Biol Chem 2004;279(49):50773-80.

12. Lee SY, Jeon HM, Ju MK, Jeong EK, Kim CH, Yoo M, et al. Dlx-2 is implicated in TGF- $\beta$-and Wnt-induced epithelialmesenchymal, glycolytic switch, and mitochondrial repression by Snail activation. Int J Oncol 2015;46(4):1768-80.
13. Wongdee K, Pandaranandaka J, Teerapornpuntakit J, Tudpor $\mathrm{K}$, Thongbunchoo J, Thongon $\mathrm{N}$, et al. Osteoblasts express claudins and tight junction-associated proteins. Histochem Cell Biol 2008;130(1):79-90.

14. Oesser S, Seifert J. Stimulation of type II collagen biosynthesis and secretion in bovine chondrocytes cultured with degraded collagen. Cell Tissue Res 2003;311(3):393-9.

15. Wang X, Li F, Fan C, Wang C, Ruan H. Effects and relationship of ERK1 and ERK2 in interleukin-1 $\beta$-induced alterations in MMP3, MMP13, type II collagen and aggrecan expression in human chondrocytes. Int J Mol Med 2011;27(4):583-9.

16. Xu C, Ji X, Harris MA, Mundy GR, Harris SE. A clonal chondrocytic cell line derived from BMP-2/T antigenexpressing transgenic mouse. In vitro Cell Dev Biol Anim 1998;34(5):359-63.

17. Watanabe H, de Caestecker MP, Yamada Y. Transcriptional cross-talk between Smad, ERK1/2, and p38 mitogen-activated protein kinase pathways regulates transforming growth factor$\beta$-induced aggrecan gene expression in chondrogenic ATDC5 cells. J Biol Chem 2001;276(17):14466-73.

18. Klatt AR, Paul-Klausch B, Klinger G, Kuhn G, Renno JH, Banerjee M, et al. A critical role for collagen II in cartilage matrix degradation: collagen II induces pro-inflammatory cytokines and MMPs in primary human chondrocytes. J Orthop Res 2009;27(1):65-70.

19. Jimenez MJ, Balbin M, Lopez JM, Alvarez J, Komori T, LopezOtin C. Collagenase 3 is a target of Cbfa1, a transcription factor of the runt gene family involved in bone formation. Mol Cell Biol 1999;19(6):4431-42.

20. Qiu M, Bulfone A, Ghattas I, Meneses JJ, Christensen L, Sharpe PT, et al. Role of the Dlx homeobox genes in proximodistal patterning of the branchial arches: mutations of Dlx-1, Dlx-2, and Dlx-1 and-2 alter morphogenesis of proximal skeletal and soft tissue structures derived from the first and second arches. Dev Biol 1997;185(2):165-84.

21. Kirsch T, Harrison G, Golub EE, Nah HD. The roles of annexins and types II and $\mathrm{X}$ collagen in matrix vesiclemediated mineralization of growth plate cartilage. J Biol Chem 2000;275(45):35577-83.

22. Xu SC, Harris MA, Rubenstein JL, Mundy GR, Harris SE. Bone morphogenetic protein-2 (BMP-2) signaling to the Col $2 \alpha 1$ gene in chondroblasts requires the homeobox gene Dlx-2. DNA Cell Biol 2001;20(6):359-65.

23. Inada $M$, Wang $\mathrm{Y}$, Byrne $\mathrm{MH}$, Rahman MU, Miyaura $\mathrm{C}$, Lopez-Otin C, et al. Critical roles for collagenase-3 (Mmp13) in development of growth plate cartilage and in endochondral ossification. Proc Natl Acad Sci USA 2004;101(49):17192-7.

This is an open access article distributed under the terms of the Creative Commons Attribution-NonCommercial-ShareAlike 3.0 License, which allows others to remix, tweak, and build upon the work non-commercially, as long as the author is credited and the new creations are licensed under the identical terms

This article was originally published in a special issue,
"Diagnostic and Therapeutic Advances in Biomedical Research
and Pharmaceutical Sciences"
Indian J Pharm Sci 2021:83(5) spl issue "55-61"

